Comentario:

\title{
¿CUÁNTOS ALBATROS DE CEJA NEGRA, THALASSARCHE MELANOPHRYS (TEMMINCK, 1828) ANIDAN EN CHILE?
}

\author{
HOW MANY BLACK BROWED ALBATROSSES THALASSARCHE \\ MELANOPHRYS (TEMMINCK, 1828) NEST IN CHILE?
}

Carlos A. Moreno ${ }^{1} \&$ Graham Robertson ${ }^{2}$

La reciente publicación en Anales del Instituto de la Patagonia del descubrimiento de una nueva colonia de albatros de ceja negra en el islote Leonard, cerca de isla Recalada en la bahía Otway por Marín \& Oehler (2007) llama la atención, no sólo por el interés que esto genera para el conocimiento de las poblaciones chilenas de esta especie, sino además porque su publicación es simultánea con otras dos publicaciones producto de un proyecto de investigación Australiano-Chileno que también hacen estimaciones del número total de albatros que anidan en Chile. Estas publicaciones estuvieron orientadas a evaluar el estado de conservación de las poblaciones chilenas, como un indicador del Plan de Acción Nacional para reducir la captura de aves en la pesquería de palangre según Decreto Supremo DS № 136 del 17 de abril 2007 (en adelante PAN-Aves marinas).

Las dos publicaciones mencionadas se refieren a una evaluación de los métodos más utilizados para conducir los censos de pares reproductores o nidos activos (Robertson et al. 2008) y consecuentemente agregar rigurosidad científica a este tipo de datos y el segundo -en una secuencia lógica (pero no bibliográfica)- hace un resumen de los censos conducidos en las colonias chilenas entre los años 2001 al 2006 usando el método que demostró ser más apropiado, como son los censos fotográficos aéreos (Robertson et al. 2007). Marín \& Oehler (2007) junto con la descripción de la nueva colonia en el islote Leonard, hacen un resumen del número de pares reproductivos del albatros de ceja negra descritos para Chile y consideran números de pares reproductivos para algunas colonias censadas con métodos diferentes del censo aéreo, respecto de los cuales los números mencionados tienen diferencias significativas respecto a los alcanzados por Robertson et al. 2007 y 2008. Consecuentemente, es deseable tener una referencia actualizada que responda la pregunta de ¿Cuántos albatros de ceja negra, Thalassarche melanophrys (Temminck, 1828) anidan en Chile? basadas en el método más preciso y más exacto como es el censo fotográfico aéreo. La respuesta a esa pregunta es, en parte, uno de los indicadores con que se medirá el éxito o fracaso de la aplicación del PAN-Aves.

\footnotetext{
${ }^{1}$ Instituto de Ecología y Evolución. Universidad Austral de Chile, Valdivia, Chile.

${ }^{2}$ Australian Antarctic Division. Kingston, Tasmania, Australia.
} 
Basándonos en Robertson et al. (2007) más el hallazgo de Marín \& Oehler (2007) los datos muestran 2,5 veces más albatros que en la tabla de Marín \& Oehler (2007) (Tabla 1).

Prácticamente todos los datos de esta tabla provienen de censos de fotografía aérea, con excepción del reciente hallazgo de Marín \& Oehler (2007). Este tipo de censo tiene la ventaja que las fotos pueden tomarse en un ángulo recto sobre las pendientes de las islas o islotes, en relación a censos en tierra o desde el mar. Ya que en estos últimos casos los ángulos son más cerrados y ocultan números importantes de nidos.

Por otra parte, las fotos digitales de alta resolución (>10 Mega píxeles) pueden ser conservadas en CD o DVD y cualquier otro autor que quiera revisar esa información puede hacerlo cumpliendo con un aspecto esencial de la rigurosidad científica: que la medición o conteo debe ser repetible. La metodología del censo aéreo está descrita en detalle en Moreno et al. (2007) y puede ser descargada de la página Web del Fondo de Investigación Pesquera (WWW. FIP.CL) en la sección de proyectos ambientales del 2006. (Proyecto FIP 2006-30).

De acuerdo a estos datos, la población chilena del albatros de ceja negra es más importante que lo que previamente se había descrito y es ligeramente más del $20 \%$ de la población mundial de albatros de esta especie, así como la población chilena del albatros de cabeza gris (Thalassarche chrysostoma Forster, 1785) es 23\% de la población mundial. Consecuentemente la responsabilidad de Chile, en la conservación de estos albatros y otros que viajan desde Nueva Zelanda y también desde Georgia del Sur (Atlántico sur) al Pacifico sur oriental, ha sido asumida seriamente al generar, poner en acción y adoptar el PAN-Aves marinas. Desde el año 2006, la pesquería demersal de bacalao de profundidad que opera entre el paralelo $47^{\circ}$ hasta el sur de la boca occidental del canal Beagle, ha disminuido la mortalidad incidental de albatros desde cerca de 2000 albatros por año en el 2002 hasta cero albatros muertos por la pesquería en las temporadas de pesca del 2006 y 2007 (Moreno et al. 2008 en prensa). Evidencias de recuperación de la población de albatros de ceja negra de la isla Gonzalo (archipiélago Diego Ramírez) en los últimos años se encuentran resumidas en Moreno et al. (2007) y muestra la actitud de pesca responsable de los palangreros industriales chilenos y de su participación activa en el PAN-Aves marinas.

La presencia de al menos 122.920 pares de albatros en Chile significa que después de la época reproductiva la población alcanza una cifra cercana a los 370.000 albatros incluyendo la cohorte del año. Como su reproducción comienza al menos a los 5 años, con toda la variabilidad ambiental que determina el éxito reproductivo se podría especular en base a estos datos que la población completa del albatros de ceja negra en Chile estaría por sobre el medio millón de individuos incluyendo la porción no reproductiva (individuos de 1 a 4 años de edad).

Sin embargo, por las extensas zonas que visitan debido a su gran amplitud de vuelos esta especie aún presenta amenazas que ameritan mayor investigación y desarrollo de la aplicación del Plan

TABLA 1. Número de pares de albatros censados en los últimos años usando censos fotográficos aéreos (con la excepción del islote Leonard).

\begin{tabular}{|c|c|c|c|c|}
\hline \multirow[t]{2}{*}{ Localidad } & \multicolumn{2}{|c|}{$\begin{array}{c}\text { Especies y № de pares } \\
\text { reproductivos }\end{array}$} & \multirow[t]{2}{*}{ Fecha de Censo } & \multirow[t]{2}{*}{ Fuente } \\
\hline & $\mathrm{ACN}^{\mathbf{1}}$ & $\mathbf{A C G}^{2}$ & & \\
\hline Isla Diego de Almagro & 15,594 & \multirow{5}{*}{8} & 25-28 Septiembre 2001 & Lawton et al. (2003) \\
\hline Islote Evangelistas & 4,670 & & 13 Octubre 2002 & Arata et al. (2003) \\
\hline Isla Ildefonso & 47,000 & & $\begin{array}{l}26 \text { Octubre } 2002 \\
4 \text { Octubre } 2006\end{array}$ & Robertson et al. (2008) \\
\hline Islote Albatros & 62 & & 4 Octubre 2006 & C. A. Moreno (unpubl.) \\
\hline Islote Leonard & 594 & & 14 Noviembre 2005 & Marín \& Oehler (2007) \\
\hline Isla Diego Ramírez & 55,000 & 17,000 & $\begin{array}{l}26 \text { Octubre-2002 Noviembre } \\
2002 \text { y } 4 \text { de Octubre } 2006\end{array}$ & Robertson et al. (2007) \\
\hline Total para Chile & 122,920 & 17,008 & & \\
\hline
\end{tabular}

1. Albatros de ceja negra y 2. Albatros de cabeza gris. 
Nacional de Acción, especialmente en aguas distantes del Pacifico sur oriental y central, donde grandes pesqueros de arrastre y palangreros pelágicos, de naciones del hemisferio norte, están ejerciendo una fuerte presión de pesca sobre el jurel y el pez espada, de la cual sólo tenemos información fragmentaria que amerita futuras acciones de conservación para el PAN-Aves marinas.

\section{LITERATURA CITADA}

Marín M. \& D. Oehler 2007. Una nueva colonia de anidamiento para el albatros de ceja negra (Thalassarche melanophrys) para Chile. Anales Instituto Patagonia (Chile) 35(1):29-33.

Moreno C.A., R.Vega, G. Robertson \& G. Luna 2007. Seguimiento del Plan de Acción de aves marinas. Informe Final proyecto FIP 2006-30, pp54 + anexos. (www.fip.cl).
Moreno C.A., R. Castro, L.J. Mújica \& P. Reyes 2008. Significant conservation benefits obtained from the use of a new fishing gear in the Chilean Industrial Patagonian Toothfish Fishery. CCAMLR Science, Vol. 15.( in press).

Robertson G., C.A. Moreno, K. Lawton, J. Arata, J. Valencia \& R. Kirkwood 2007. The status of black-browed and grey -headed albatrosses at Diego Ramirez Archipelago, Chile. Emu, 107 (3): 239-244.

Robertson G., C.A. Moreno, K.Lawton, R. Kirkwood $\&$ J. Valencia 2008. Comparison of census methods for black-browed albatross breeding at the Ildefonso Archipelago, Chile. Polar Biology 32(2): 153-162. 
C. MORENO et al. 